\title{
Minimal differential lateral acceleration configurations for starshade stationkeeping in exoplanet direct imaging
}

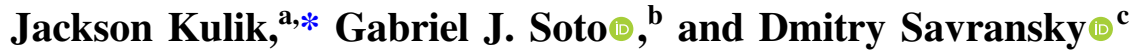 \\ ${ }^{a}$ Cornell University, Center for Applied Mathematics, Ithaca, New York, United States \\ ${ }^{b}$ University of Wisconsin-Madison, Department of Engineering Physics, Madison, \\ Wisconsin, United States \\ ${ }^{\mathrm{c} C}$ Cornell University, Department of Mechanical and Aerospace Engineering, Ithaca, \\ New York, United States
}

\begin{abstract}
Exoplanet imaging missions utilizing an external occulter (starshade) for starlight suppression require precise alignment between the telescope and starshade, necessitating maintenance of the starshade orbit during observations. Differential lateral acceleration between the two spacecraft serves as a proxy for fuel use and number of required thruster firings, which may interrupt the observation. Comparison against results from high fidelity simulations of stationkeeping validates the use of this easy-to-compute proxy. Among starshade positions constrained to the surface of a sphere centered about the telescope, minima of differential lateral acceleration lie on a great circle and its corresponding poles. We present a closed expression for telescope to star vectors requiring minimal stationkeeping for observation from a telescope at an arbitrary position. This proxy metric along with analytical knowledge of optimal observations facilitates computationally efficient design of exoplanet imaging missions employing a starshade. ( $)$ The Authors. Published by SPIE under a Creative Commons Attribution 4.0 International License. Distribution or reproduction of this work in whole or in part requires full attribution of the original publication, including its DOI. [DOI: 10.1117/1.JATIS.8.1.017003]
\end{abstract}

Keywords: starshade; exoplanet; direct imaging; stationkeeping.

Paper 21139G received Oct. 29, 2021; accepted for publication Feb. 22, 2022; published online Mar. 8, 2022.

\section{Introduction}

Given a space-based telescope, nominally along an Earth-Sun L2 Halo orbit, a starshade located at a fixed distance from the telescope, and a target star, we analyze the costs and timing metrics associated with maintaining the starshade in a nominal position, within some tolerance, to block out light from the given star. Our work is in the same vein as previous analyses from Refs. 1 to 3; however, we take a more approximate analytical approach with the goal of furthering understanding and speeding up computation. We model the use of impulsive burns to maintain the starshade within a 1-m lateral tolerance of the line of sight between telescope and target star. ${ }^{4}$ Fuel costs and number of stationkeeping maneuvers required during an observation can serve as important considerations in deciding between target stars for observation. These stationkeeping metrics may be used in an objective function that would ideally evaluate quickly as part of a larger scheduling problem optimization such as those described in Refs. 5 to 8.

A stationkeeping strategy that maximizes time between impulsive burns is given by Ref. 1 . This strategy for deadbanding assumes constant differential acceleration between telescope and starshade. The authors of Ref. 3 further explored this strategy with or without axial stationkeeping, analyzing fuel use and observational efficiency while also considering optical keep out zones. Both papers numerically solved ordinary differential equations (ODEs) with event driven application of the impulses to find stationkeeping metrics. This is a computationally expensive operation, and it would not be desirable to carry out these integrations as part of a larger mission design optimization process, motivating the need for a fast method to approximate the

*Address all correspondence to Jackson Kulik, jpk258@ cornell.edu 
stationkeeping metrics and a comparison of accuracy between metrics obtained by approximation or numerical integration.

We summarize the work of Ref. 1 and then compare their analytical strategy under ideal conditions against numerical results from Ref. 3. This serves to validate the use of the inexpensive analytical model for stationkeeping. From there, we continue the linear analysis in Sec. 6 and Appendix C of Ref. 1 on the lateral differential acceleration, focusing on the locations of its minima. We present a geometric picture of differential lateral acceleration and an analytical expression to approximate locations associated with low stationkeeping costs. Among starshade positions constrained to the surface of a sphere centered about the telescope, minima of differential lateral acceleration lie on a great circle and its corresponding poles. This observation along with an analytical expression for the minima could be used as a heuristic to reduce the design space for scheduling of an exoplanet imaging mission. Our focus on minima of differential lateral acceleration for starshade formation flying purposes stands in contrast to the study of minima of axial/radial differential acceleration for general purpose formation flying as seen in Refs. 9 and 10. This analysis of easily computable formation flying metrics and optimal configurations under them contributes to recent efforts geared toward analyzing requirements for and eventually planning starshade missions. ${ }^{1,11-13}$

\section{Analytical Model of Stationkeeping Metrics}

We consider the inertial accelerations of the telescope $\mathbf{a}_{t}$ and starshade $\mathbf{a}_{s}$ with respect to the inertially nonaccelerating barycenter at an initial epoch and then assume that the differences in inertial accelerations between satellites remain constant over the course of the observation. In the inertial frame, the direction of the relative position vector $\mathbf{r}_{\text {rel }}$ between the starshade at $\mathbf{r}_{s}$ and telescope at $\mathbf{r}_{t}$ should remain constant to preserve alignment of the telescope, starshade, and target. For convenience, consider an inertial frame which happens to be aligned with the circular restricted three body problem (CR3BP) rotating frame at the initial epoch and in units of AU. The frame is shown in Fig. 1 with the Earth-Sun barycenter located at the origin. Consider all accelerations from this point forward as inertial.

In the inertial reference frame, the acceleration of satellite $j=t, s$ from two bodies is given as

$$
\begin{gathered}
\mathbf{a}_{j}=\mathbf{a}_{j, 1}+\mathbf{a}_{j, 2}, \\
\mathbf{a}_{j, 1}=\frac{(\mu-1)\left(\mathbf{r}_{j}+\mu \hat{\mathbf{i}}\right)}{\left\|\mathbf{r}_{j}+\mu \hat{\mathbf{i}}\right\|^{3}}, \\
\mathbf{a}_{j, 2}=\frac{-\mu\left(\mathbf{r}_{j}+(\mu-1) \hat{\mathbf{i}}\right)}{\left\|\mathbf{r}_{j}+(\mu-1) \hat{\mathbf{i}}\right\|^{3}},
\end{gathered}
$$

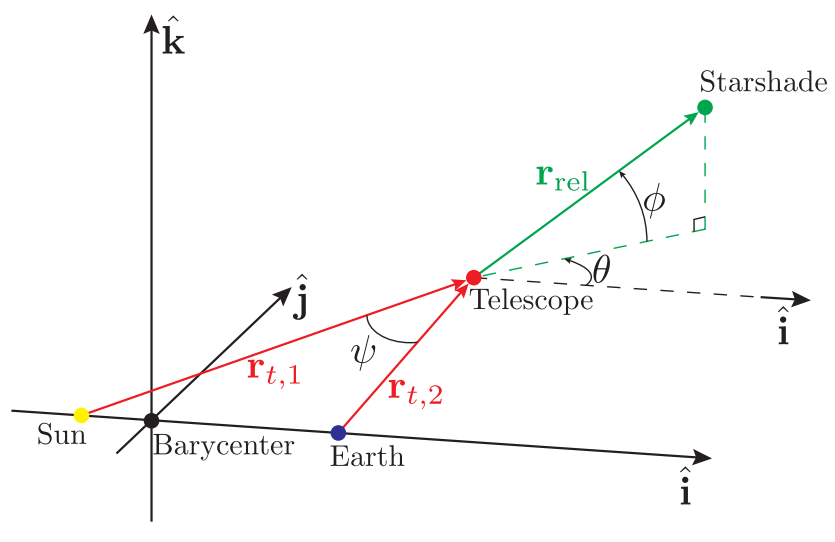

Fig. 1 The coordinate frame employed in this paper with relevant bodies and angles depicted. 
where $\mu$ is the mass parameter, subscript 1 refers to the primary body (the Sun), 2 refers to the secondary body (the Earth), $\hat{\mathbf{i}}$ is the unit vector in the Sun-Earth direction, and $\mathbf{r}$ refers to position. The Sun is assumed to be at $-\mu \hat{\mathbf{i}}$ and the Earth at $(1-\mu) \hat{\mathbf{i}}$.

The differential acceleration between starshade and telescope is given by the difference in the inertial second time derivatives of position

$$
\delta \mathbf{a}=\mathbf{a}_{s}-\mathbf{a}_{t}=\ddot{\mathbf{r}}_{s}-\ddot{\mathbf{r}}_{t}
$$

Starshade position is nominally given as

$$
\mathbf{r}_{s}=\mathbf{r}_{t}+R(\cos \phi \cos \theta \hat{\mathbf{i}}+\cos \phi \sin \theta \hat{\mathbf{j}}+\sin \phi \hat{\mathbf{k}}),
$$

where, as shown in Fig. 1, $R=\left\|\mathbf{r}_{\text {rel }}\right\|$ is the nominal telescope-starshade separation distance; $\theta$, the ecliptic longitude, is the in-plane angle of the star from the Sun-Earth vector at the starting epoch; and $\phi$, the ecliptic latitude, is the out-of-plane angle measured from the ecliptic plane as measured with respect to the starshade location.

Consider the axial and lateral components of the differential acceleration, where axial denotes the direction of the telescope line of sight to the target star, and lateral denotes the component orthogonal to the line of sight. The lateral differential acceleration magnitude is given as

$$
\delta a_{l}=\left\|\delta \mathbf{a}-\left(\delta \mathbf{a} \cdot \hat{\mathbf{r}}_{\text {rel }}\right) \hat{\mathbf{r}}_{\text {rel }}\right\|,
$$

where

$$
\hat{\mathbf{r}}_{\mathrm{rel}}=\cos \phi \cos \theta \hat{\mathbf{i}}+\cos \phi \sin \theta \hat{\mathbf{j}}+\sin \phi \hat{\mathbf{k}}
$$

The starshade has a nominal relative position with respect to the telescope and a lateral tolerance $r_{\text {tol }}$ from this position. We consider lateral stationkeeping, disregarding axial stationkeeping, because axial tolerances are typically larger than the distances traversed during a typical observation. An axial tolerance of $250 \mathrm{~km}$ will be violated only after at least 4 days without axial stationkeeping according to Ref. 3.

In the optimal strategy outlined in Ref. 1, the starshade begins some nominal axial distance from the telescope and at the edge of the $r_{\text {tol }}$ lateral tolerance disc in the direction of lateral relative acceleration. The starshade is initialized with lateral relative velocity chosen in the opposite direction of lateral relative acceleration. The magnitude of the velocity is chosen such that the starshade will reach the exact opposite end of the disk with radius given by the lateral tolerance and then come back down to its initial position under the constant lateral acceleration. At this point, the starshade applies an impulsive burn to repeat the process. The resulting stationkeeping metrics are given as follows:

$$
\begin{gathered}
T=4 \sqrt{\frac{r_{\mathrm{tol}}}{\delta a_{l}}} \\
N=\text { floor }\left(\frac{\tau_{\mathrm{obs}} \sqrt{\delta a_{l}}}{4 \sqrt{r_{\mathrm{tol}}}}\right), \\
\Delta v=4 N \sqrt{\delta a_{l} r_{\mathrm{tol}}},
\end{gathered}
$$

where $\tau_{\text {obs }}$ is the overall observation/stationkeeping time, $T$ is the amount of time between impulsive burns, $N$ is the number of burns after the initial epoch (not counting the initialization of the starshade), and $\Delta v$ is the cost in terms of impulsive velocity changes to perform the stationkeeping. These results were presented in nondimensional form in more generality for arbitrary initialization positions on the tolerance disk. ${ }^{1}$ In Ref. 1, the nondimensional time units (TU) are $\sqrt{r_{\text {tol }} / \delta a_{l}}$, and the longest possible time between maneuvers is 4 . The initial nondimensional velocity (scaled by $\sqrt{r_{\text {tol }} \delta a_{l}}$ ) is 2 before the starshade begins to drift from the bottom of the disk and negative 2 when it reaches the original location. Thus each maneuver requires four nondimensional units of delta-v. This yields Eqs. (7) to (9) in dimensional form. Notice that $\Delta v$ 
is largely independent of the stationkeeping tolerance and varies as $\tau_{\mathrm{obs}} \delta a_{l}$, the product of observation time and acceleration magnitude. We can obtain various other metrics as a function of Eqs. (7) to (9) as in Ref. 3.

While it is possible that the delta-v for stationkeeping could be small compared to the delta-v required for slewing, minimizing differential lateral acceleration is important for the following reasons. The delta-v associated with stationkeeping most likely cannot come from constant thrust electric propulsion to avoid sunlight scattering off continuously produced plumes saturating detectors on the telescope. ${ }^{1}$ So, stationkeeping delta-v requirements are important independent of their magnitude relative to starshade slewing delta-v requirements between target stars which can be accomplished by electric propulsion. In addition, differential lateral acceleration is important in the timing between stationkeeping maneuvers given a fixed stationkeeping tolerance. This means that differential lateral acceleration in the telescope to starshade configuration determines the percentage of time that can be dedicated to observation and generation of science data. Since differential lateral acceleration influences chemical propulsion delta-v requirements and observational efficiency, we will study it as a function of telescope and starshade configurations.

The metrics presented in Eqs. (7) to (9) are relatively simple to evaluate compared to the numerical solution of an ODE that depends on repeatedly evaluating the differential acceleration. As each of these metrics depends simply on differential lateral acceleration, we study this quantity as a proxy for all the other metrics. A plot of lateral differential acceleration is given in Fig. 8 of Ref. 1. However, only a small region around a maximum is depicted as the worst case scenarios were the primary focus of the work. The overall structure of the differential acceleration is not given, and in particular, the minima are not shown. Figure 2 sheds some light on the structure of differential lateral acceleration and resembles the color plots seen in Fig. 9 of Ref. 3, taking the plots from the plane to the sphere. Looking at differential lateral acceleration as calculated by Eq. (6) projected on the unit sphere centered about the telescope, we can understand that the minima of lateral acceleration magnitude lie on a great circle and its corresponding poles. In Fig. 2, the telescope is assumed to be at $(x, y, z)=(1+2.5 / 150,0,1 / 150)$. The starshade is assumed to be $R=100,000 \mathrm{~km}$ away from the telescope. The existence of the great circle and poles can be explained by the rejection of the axial component of acceleration. Figure 3 shows a plot of the differential acceleration vector field, demonstrating that the vector field is orthogonal to the sphere in the axial or antiaxial direction along the same great circle and at the two corresponding poles. More specifically, the vector field in Fig. 3 points outward on the far left and right and then inward toward the origin along the circle lying between the two extreme points. We also depict the cosine of the angle between relative position and differential acceleration in Fig. 3. This gives a less intuitive but more clear representation of the direction of the differential acceleration vector. Values of $-1,0,1$ correspond to differential acceleration that is purely antiaxial, lateral, and axial, respectively.
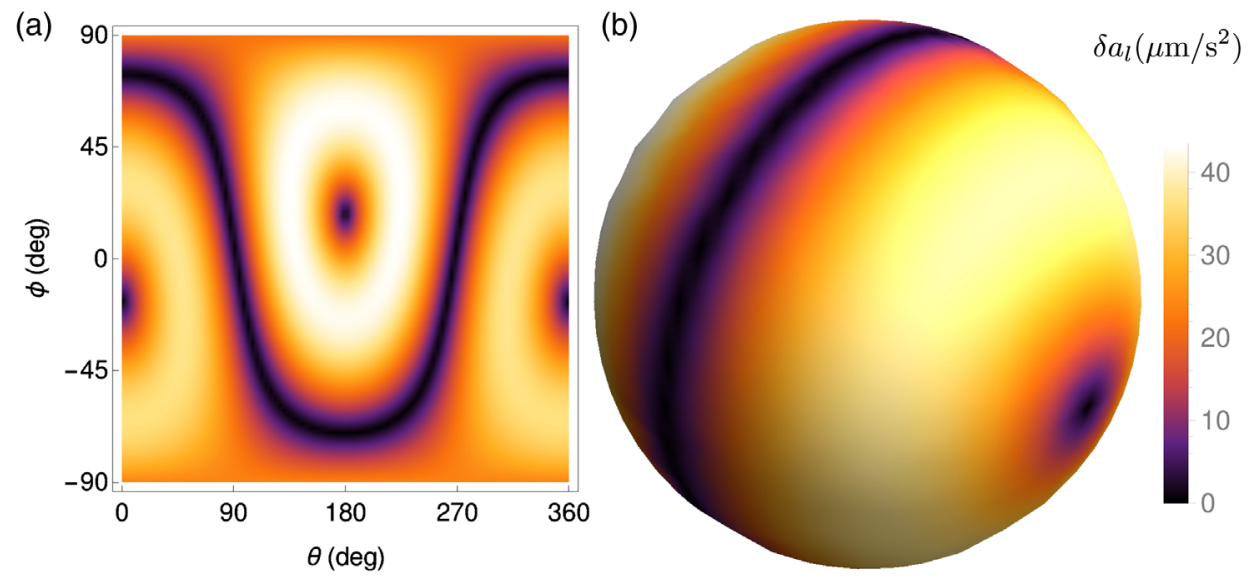

Fig. 2 The differential lateral acceleration in $\mu \mathrm{m} / \mathrm{s}^{2}$ as a function of target coordinates. (a) The density plot is accompanied by (b) its projection onto the unit sphere. 

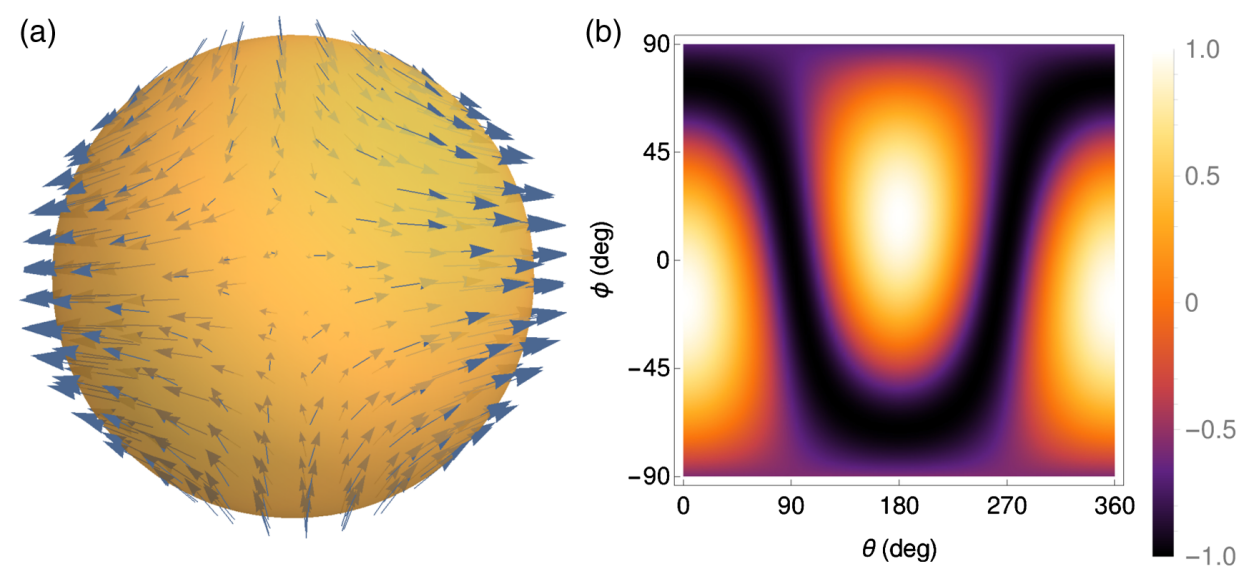

Fig. 3 (a) The differential acceleration vector field along the unit sphere about the telescope. (b) The cosine of the angle between relative position and differential acceleration.
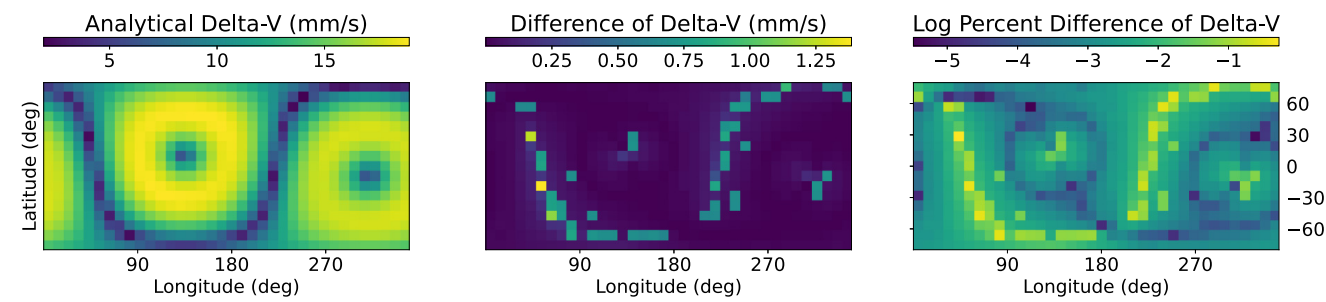

Fig. 4 Comparison between numerical and analytical models of the average delta-v to perform a single stationkeeping impulse in $\mathrm{mm} / \mathrm{s}$ during an observation at 340 days from the start of the reference halo orbit. The numerically derived average delta- $v$ is not pictured as it is visually identical to the analytical delta-v.

Figure 4 demonstrates the similarity between the numerically computed and analytically computed $\Delta v$ metric for a telescope 340 days from initialization of the halo orbit. Analytical computation is described above, while the numerical computation is carried out with only gravitational effects averaging the delta-v for burns taking place over a 6-h period as in Ref. 3. The average delta- $v$ is required here since the differential lateral acceleration is only nearly constant. We see that the two metrics agree very well except along the minima of $\Delta v$, where they differ by up to half of the analytical value. The effects of nonconstant differential lateral acceleration are a plausible explanation of these differences. It is reasonable to believe that at these minima induced by the direction of differential acceleration, small changes in differential acceleration direction would have greater effects than at other locations. However, overall structure from the two approaches is the same, with minima in the nearly the same locations.

\section{Approximate Minima of Lateral Acceleration}

The location of one of the poles shown in Fig. 2 can be computed by numerical minimization with a method such as conjugate gradient on the differential lateral acceleration as a function of pointing angles of the telescope. We give the angular locations of the pole in Fig. 6 as the telescope moves along the 6-month reference halo orbit depicted in Fig. 5. Nondimensional TU are used with one revolution of the Earth around the Sun corresponding to $2 \pi$ TU. Since the pole stays in a fairly narrow region for the given halo orbit, and the location is considered in the rotating Sun-Earth frame, the great circle corresponding to the pole sweeps out the entire celestial sphere in inertial space. This means that for any star there is some time of year in which it lies on the great circle of minimal differential lateral acceleration.

Employing a poor initial guess can cause numerical methods to identify minima along the great circle rather than the pole. Serving as an initial guess for numerical minimization, the pole's 


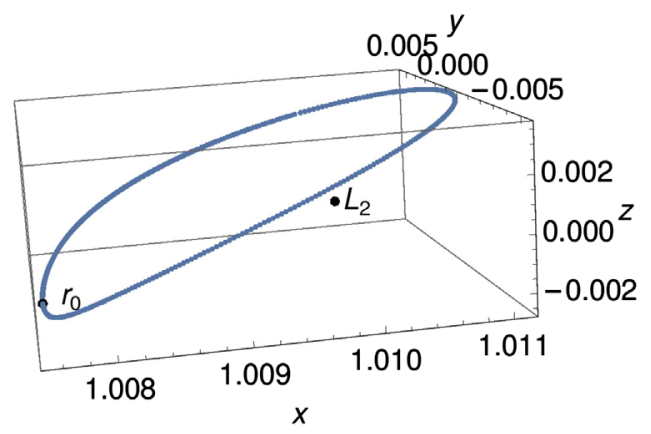

Fig. 5 The 6-month period reference halo orbit, with the initial position labeled in canonical units of the CR3BP. The initial out of plane position of this orbit is $\sim 418,000 \mathrm{~km}$.

(a)

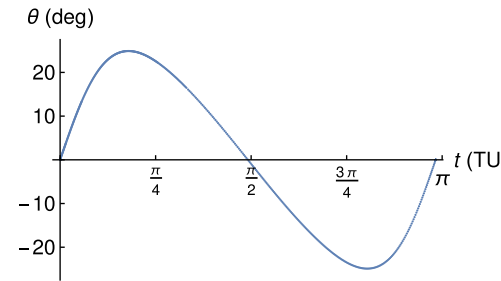

(b)

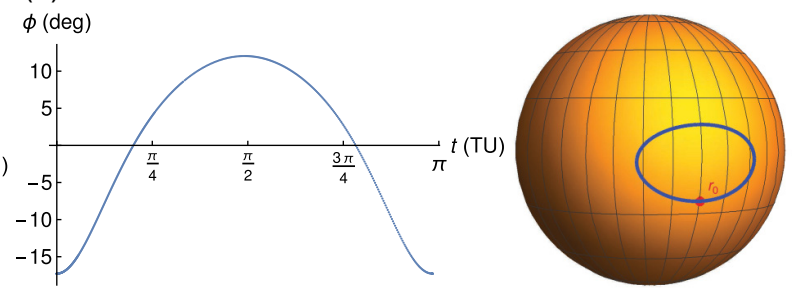

Fig. 6 Pole location as the telescope moves along the reference halo orbit trajectory in terms of ecliptic longitude, latitude, and location on the unit sphere.

location can be approximated quickly from the eigenvectors of the Jacobian matrix of the inertial acceleration vector of the telescope $D \mathbf{a}\left(\mathbf{r}_{t}\right)=\left(\partial \mathbf{a}\left(\mathbf{r}_{t}\right) / \partial \mathbf{r}_{t}\right)$ :

$$
\delta \mathbf{a}\left(\mathbf{r}_{t}\right)=\mathbf{a}\left(\mathbf{r}_{t}+\mathbf{r}_{\mathrm{rel}}\right)-\mathbf{a}\left(\mathbf{r}_{t}\right) \approx D \mathbf{a}\left(\mathbf{r}_{t}\right) \cdot \mathbf{r}_{\mathrm{rel}} .
$$

Equation (10) demonstrates that the differential acceleration will be completely axial in the corresponding linearized system precisely when the differential acceleration $\delta \mathbf{a}\left(\mathbf{r}_{t}\right)$ is parallel or antiparallel to the offset vector between telescope and starshade $\mathbf{r}_{\text {rel }}$. This is the case when $\mathbf{r}_{\text {rel }}$ is an eigenvector of $D \mathbf{a}\left(\mathbf{r}_{t}\right)$. Given the structure of the vector field in Fig. 3, the eigenvector corresponding to the pole will be the one corresponding to a positive eigenvalue. The other two eigenvectors correspond to points on the great circle. For telescope locations along the reference halo orbit, the difference in angular position of the pole as computed by the numerical method in Fig. 6, and the linear approximation and eigenvector method is on the order of a degree for $R=100,000 \mathrm{~km}$, scaling linearly with $R$.

Even with the relatively easy to compute eigenvector interpretation, we still seek an analytical expression for the pole locations. We begin by approximating the differential acceleration by a binomial series as in Ref. 14, [p. 391]:

$$
\begin{gathered}
\delta \mathbf{a}=\delta \mathbf{a}_{1}+\delta \mathbf{a}_{2}, \\
\delta \mathbf{a}_{j} \approx \frac{a_{t, j} \mathbf{r}_{\mathrm{rel}}}{r_{t, j}}-\frac{3 \mathbf{a}_{t, j}}{r_{t, j}}\left(\hat{\mathbf{a}}_{t, j} \cdot \mathbf{r}_{\mathrm{rel}}\right), \\
\delta a_{j, l} \approx \frac{3 a_{t, j} \sin \theta_{j}}{r_{t, j}}\left(\hat{\mathbf{a}}_{t, j} \cdot \mathbf{r}_{\mathrm{rel}}\right)=\frac{3 R a_{t, j} \sin \theta_{j} \cos \theta_{j}}{r_{t, j}},
\end{gathered}
$$

where $j$ denotes the relevant body ( 1 for Sun and 2 for Earth), the subscript $l$ denotes the lateral component of the acceleration, and $\theta_{j}$ the angle between $\mathbf{r}_{\text {rel }}$ and $\mathbf{a}_{t, j}$ or equivalently between $\mathbf{r}_{\text {rel }}$ and $-\mathbf{r}_{t, j}$. We will use $a, r$ to denote the norms $\|\mathbf{a}\|,\|\mathbf{r}\|$, respectively. To find configurations that induce axial differential acceleration, we find values of $\mathbf{r}_{\text {rel }}$ that balance $\delta \mathbf{a}_{1}$ against $\delta \mathbf{a}_{2}$ so their sum is in the direction of $\mathbf{r}_{\text {rel }}$. This corresponds to when their lateral components $\delta a_{1, l}$ and $\delta a_{2, l}$ 


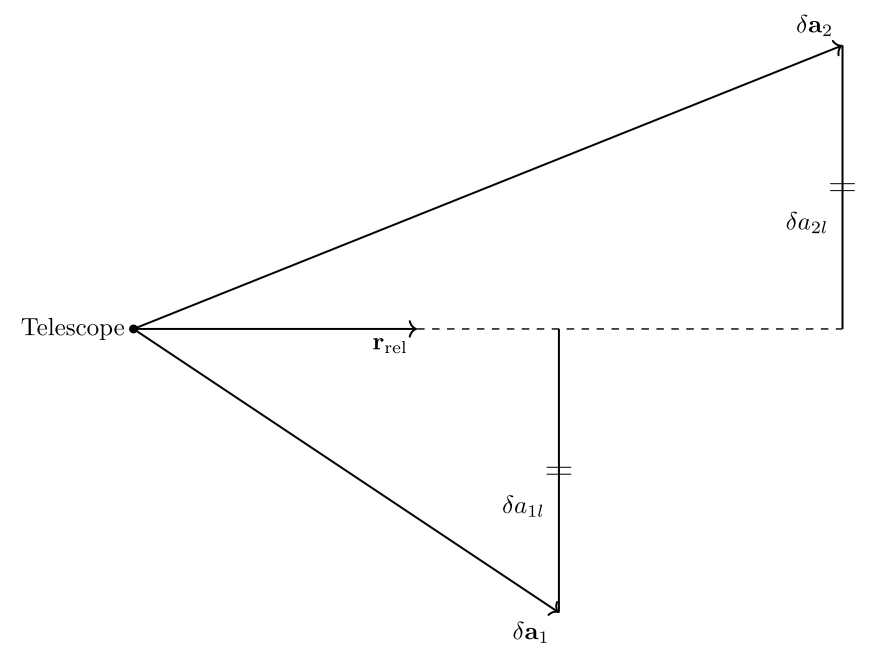

Fig. 7 The desired orientation of the telescope to starshade vector $\left(\mathbf{r}_{\text {rel }}\right)$ relative to differential acceleration vectors from Sun and Earth gravity $\left(\delta \mathbf{a}_{1}, \delta \mathbf{a}_{2}\right)$.

are equal as shown in Fig. 7. Since the first term in the approximate differential acceleration from each body is in the direction of $\mathbf{r}_{\text {rel }}$, the problem reduces to balancing the second terms of Eq. (12) so the contribution from the Sun and the Earth cancel each other out. After dividing by common terms, differential axial acceleration is equivalent to the following conditions:

$$
\begin{gathered}
\delta a_{1, l}=\delta a_{2, l} \quad \Leftrightarrow \\
0=\frac{a_{t, 1}}{r_{t, 1}} \cos \theta_{1} \sin \theta_{1}-\frac{a_{t, 2}}{r_{t, 2}} \cos \theta_{2} \sin \theta_{2} \quad \Leftrightarrow \\
0=\frac{a_{t, 1}}{r_{t, 1}} \sin 2 \theta_{1}-\frac{a_{t, 2}}{r_{t, 2}} \sin 2 \theta_{2} .
\end{gathered}
$$

Defining $\psi=\theta_{1}+\theta_{2}$ as the angle between the vectors from the telescope to the two bodies, such that

$$
\psi=\arccos \left(\frac{r_{t, 1}^{2}+r_{t, 2}^{2}-1}{2 r_{t, 1} r_{t, 2}}\right)
$$

Equation (16) becomes

$$
0=\frac{a_{t, 1}}{r_{t, 1}} \sin 2 \theta_{1}-\frac{a_{t, 2}}{r_{t, 2}} \sin \left(2 \psi-2 \theta_{1}\right) .
$$

Expanding the trigonometric term in Eq. (18), we obtain

$$
\theta_{1}=\frac{1}{2} \arctan \left(\frac{\sin (2 \psi) a_{2} / r_{2}}{a_{1} / r_{1}+\cos (2 \psi) a_{2} / r_{2}}\right)
$$

dropping the subscript $t$ for readability.

To summarize, the location of one of the poles is in the plane of the Earth, Sun, and telescope, situated between the telescope to Earth vector $\left(\mathbf{r}_{\mathbf{t}, 2}\right)$ and the telescope to Sun vector $\left(\mathbf{r}_{t, 1}\right), \theta_{1}$ in angle away from the telescope to Sun vector, $\mathbf{r}_{t, 1}$. One may rotate a unit vector in the direction from the telescope to the Sun, $\hat{\mathbf{r}}_{t, 1}$, about the $-z_{t} \hat{\mathbf{j}}+y_{t} \hat{\mathbf{k}}$ axis by $\theta_{1}$ to obtain a unit vector in the direction of the pole. Note that the results of this approach are identical to the eigenvector approach as well as numerical minimization of the approximate linear differential lateral acceleration. Given the location of a single pole that we have described, the other pole and the corresponding great circle are also known. 
(a)

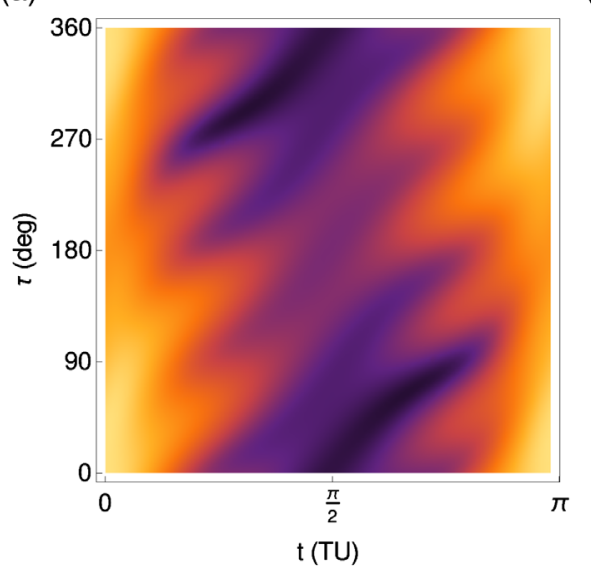

(b)

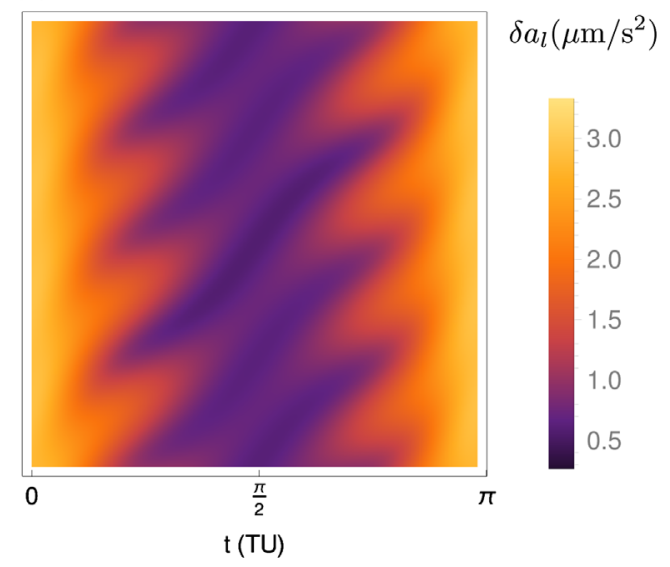

Fig. 8 Differential lateral acceleration in $\mu \mathrm{m} / \mathrm{s}^{2}$ is depicted for a starshade on a $100,000 \mathrm{~km}$ radius great circle about a telescope moving along the reference halo orbit. (a) Exact and (b) approximate pole positions describe the great circle.

The great circle is of particular interest since it is a higher dimensional object than the two poles. Many more stars will be easily observable along the great circle than on or near the poles. As a result, it becomes important to quantify how low the differential lateral acceleration is along the great circle. We employ Eq. (19) to find a unit vector in the approximate direction of the pole and potentially improve on this by numerical minimization of the differential lateral acceleration. Upon obtaining the approximate or the exact pole location, the corresponding great circle consists of all of the unit vectors orthogonal to the pole vector. We may parameterize this great circle arbitrarily with a parameter $\tau$, the angle in radians from some reference vector along the great circle. We employed the cross product of $\hat{\mathbf{i}}$ and the pole vector as our reference vector on the great circle. To obtain the entire great circle, we rotated the reference vector by $\tau$ about the pole vector. Figure 8 shows the differential lateral acceleration along the great circles corresponding to the exact and approximate pole locations at each point along the halo orbit. Position of the telescope along the halo orbit is given along the $x$ axis by the time $t$ from the initial epoch when the telescope is located at $\mathbf{r}_{0}$ as shown in Fig. 5. At each of these values of $t$, the position of the telescope leads to a direction for a pole given by Eq. (19), which correspond to the values in Fig. 6. Each time $t$ maps to a pole location, which in turn maps to a single corresponding great circle. Position along the great circle is given on the $y$ axis by $\tau$, the arbitrarily chosen phasing parameter. Note that time values near 0 and $\pi$ lead to generally higher differential lateral accelerations as the two satellites are closer to the Earth at this point in the halo orbit. One can see that as the telescope moves along its halo orbit, even at its closest point to Earth, the differential lateral acceleration along the corresponding great circle is consistently one to two orders of magnitude below the highest values shown in Fig. 2. Using Eqs. (7) to (9), with a lateral position tolerance of $1 \mathrm{~m}$, this translates to a difference between six stationkeeping maneuvers per hour in the worst case and fewer than one interruption per hour for observations along the great circle. Considering both the exact or approximate great circle effectively determines convenient choices of stars for more fuel efficient and less frequently interrupted observations.

\section{Conclusion}

We have explored trends involving station keeping metrics for observation of exoplanets with a telescope and starshade pair. Differential lateral acceleration proved to be an effective proxy for metrics such as time between interruptions for starshade orbit maintenance and delta-v. Using this metric as a proxy enables mission design software to estimate stationkeeping costs with a simple analytical calculation rather than a numerical integration as has been previously done. 
In addition, we have explained trends in stationkeeping costs through an analytical approach. For a fixed position of the telescope, differential lateral acceleration as a function of the line-ofsight vector from telescope to target star proved to possess minima along two poles and their corresponding great circle. We derived an analytical approximation for the location of these minima and demonstrated that for a design reference mission, observing stars along the great circle yields magnitudes of differential lateral acceleration that are one or two orders of magnitude lower than if the target were chosen indiscriminately. We advocate cognizance of this great circle of easy to observe targets during design of exoplanet missions. By considering targets as they cross or at least approach this great circle, one may potentially pare down the large design space for scheduling problems related to exoplanet imaging missions. While keepout zones and other constraints may make it impossible to take advantage of configurations along the minima of differential acceleration at some points, all stars will lie on the great circle at some point in the year, making the great circle of minimal differential lateral acceleration a potentially useful design tool.

\section{Acknowledgments}

This work was supported by NASA JPL SURP Grant RSA No. 1644208.

\section{References}

1. T. L. Flinois et al., "Starshade formation flying ii: formation control," J. Astron. Telesc. Instrum. Syst. 6(2), 029001 (2020).

2. D. Sirbu, C. V. Karsten, and N. J. Kasdin, "Dynamical performance for science-mode stationkeeping with an external occulter," Proc. SPIE 7731, 773152 (2010).

3. G. J. Soto, D. Savransky, and R. Morgan, "Analytical model for starshade formation flying with applications to exoplanet direct imaging observation scheduling," J. Astron. Telesc. Instrum. Syst. 7(2), 021209 (2021).

4. S. Seager et al., "The Exo-S probe class starshade mission," Proc. SPIE 9605, 96050W (2015).

5. D. R. Keithly et al., "Optimal scheduling of exoplanet direct imaging single-visit observations of a blind search survey," J. Astron. Telesc. Instrum. Syst. 6(2), 027001 (2020).

6. W. D. Sanchez, "Towards fuel-efficient formation flying of an observatory and external occulter at Sun-Earth L2," PhD thesis, Massachusetts Institute of Technology (2020).

7. G. Soto et al., "Optimal starshade observation scheduling," Proc. SPIE 10698, 106984M (2018).

8. G. J. Soto et al., "Parameterizing the search space of starshade fuel costs for optimal observation schedules," J. Guidance Control Dyn. 42(12), 2671-2676 (2019).

9. F. Salazar et al., "Zero, minimum and maximum relative radial acceleration for planar formation flight dynamics near triangular libration points in the earth-moon system," Adv. Space Res. 54(9), 1838-1857 (2014).

10. F. J. T. Salazar et al., "Zero drift regions and control strategies to keep satellite in formation around triangular libration point in the restricted sun-earth-moon scenario," Adv. Space Res. 56(7), 1502-1518 (2015).

11. M. Bottom et al., "Starshade formation flying i: optical sensing," J. Astron. Telesc. Instrum. Syst. 6(1), 015003 (2020).

12. L. M. Palacios, A. Harness, and N. J. Kasdin, "Hardware-in-the-loop testing of formation flying control and sensing algorithms for starshade missions," Acta Astron. 171, 97-105 (2020).

13. P. Willems and D. Lisman, "NASA'S starshade technology development activity," J. Astron. Telesc. Instrum. Syst. 7(2), 021203 (2021).

14. D. A. Vallado, Fundamentals of Astrodynamics and Applications, Vol. 4, Springer Science \& Business Media (2001).

Jackson Kulik is a PhD student at the Cornell Center for Applied Mathematics and an NDSEG fellowship recipient. His research focuses on the application of dynamical systems theory to 
enable fast and accurate computation in guidance, navigation, and control problems related to satellite formation flight.

Gabriel J. Soto received his $\mathrm{PhD}$ in aerospace engineering from Cornell University in December 2020. His research has focused on fuel cost heuristics, formation flying, and trajectory design of spacecraft-notably starshades-in astrophysics and exoplanet direct imaging missions. He is now a postdoctoral research associate at the University of Wisconsin-Madison studying the optimization of integrated nuclear and renewable energy system design and operation.

Dmitry Savransky is an associate professor in the Sibley School of Mechanical and Aerospace Engineering and Department of Astronomy at Cornell University, where he runs the Space Imaging and Optical Systems Laboratory. His research focuses on the application of estimation and computer vision techniques to control and autonomously operate advanced optical systems and on the design and optimization of astrophysics-focused space missions. 